\title{
Benefits of photonic structuring on perovskite solar cells using opal-like layers
}

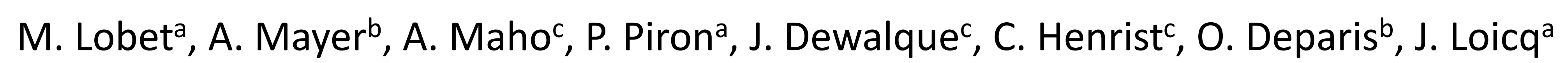

\author{
aCentre Spatial de Liege, STAR institute, University of Liege, Belgium \\ bSolid-State Physics Laboratory, University of Namur, Belgium \\ CESAM-GREENMAT, University of Liege, Belgium
}

Abstract

Perovskite solar cells (PSC) have been under the spotlight of the photovoltaics community since the past decade due notably to high instrinsic absorption of pervoskite. However, little is known on the impact of structuring the active material using photonic crystal layers. We present here numerical simulations showing the effect of photonic crystal structuring on the integrated quantum efficiency of perovskite solar cells. The photo-active layer is structured using opal-like perovskite layers (monolayers, bilayers or trilayers) made of perovskite (full or truncated) spheres, including hybrid uniform/structured layers, embedded in a $\mathrm{TiO}_{2}$ matrix. Fano resonances are exploited in order to enhance the absorption, especially near the electronic bandgap of perovskite material. The excitation of quasiguided modes inside the absorbing spheres increases the integrated quantum efficiency and the photonic enhancement factor. A genetic algorithm approach allows us to determine the optimum structure among more than $1.410^{9}$ potential combinations. These numerical results of the benefits of photonic structuring on perovskite solar cells are also compared to experimental studies on selected configurations of perovskite solar cells.

\section{Photonic structures and materials}

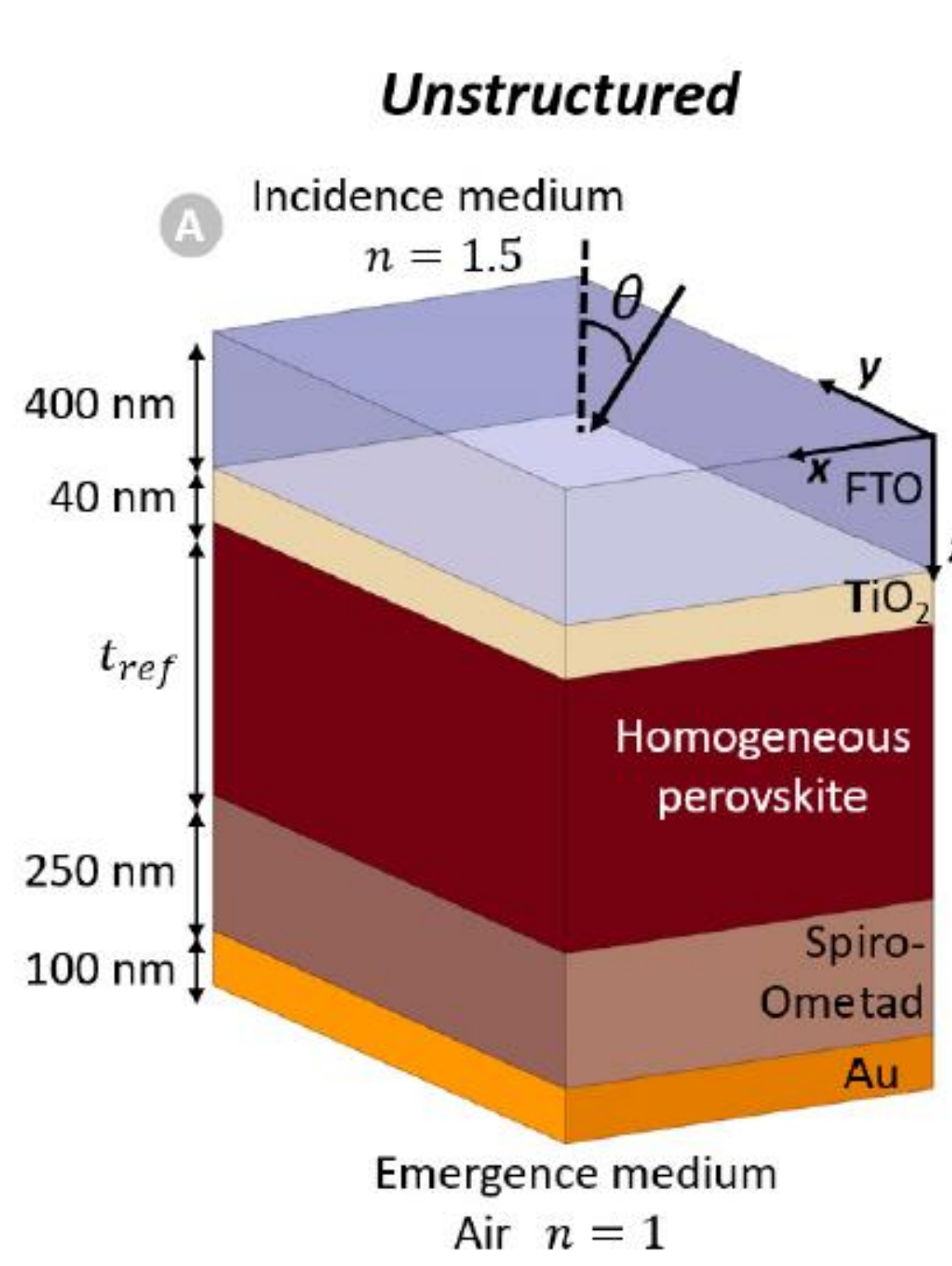

Role of the different layers

- FTO: transparent conducting electrode

- $\mathrm{TiO}_{2}$ : hole blocking layer

Perovskite $\mathrm{CH}_{3} \mathrm{NH}_{3} \mathrm{Pbl}_{3}$ layer: photo-active laye

(unstructured vs 3D structuration)

Spiro-Ometad: hole transporting layer

Au: counter-electrode and back reflector

Goals (GA)

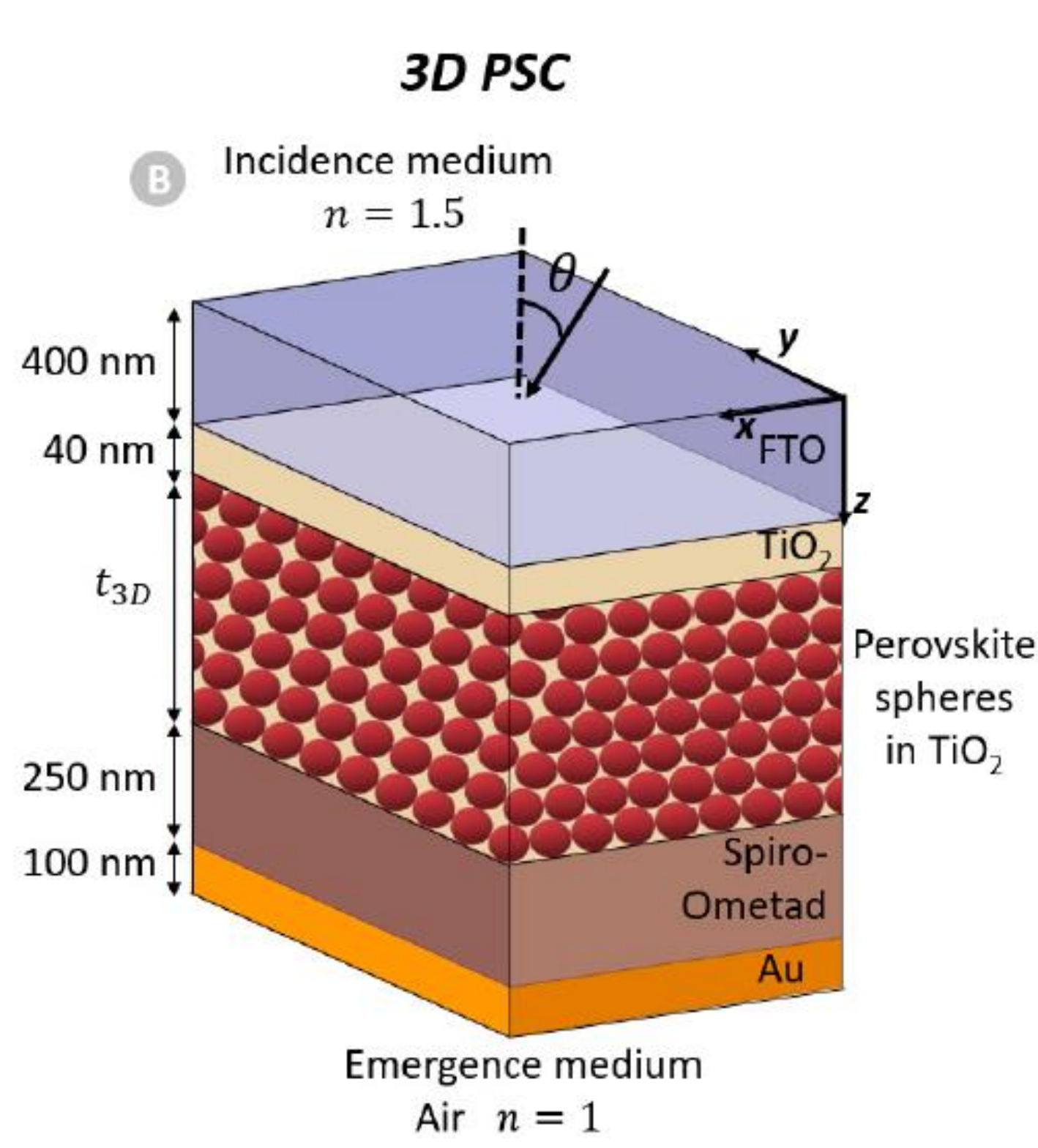

$\checkmark$ Compare the effect of $3 D$ structuring vs unstructured photo-active perovskite layer on the integrated quantum efficiency (IQE $\eta$ ) Optimize absorption using a Genetic Algorithm

Confirm the numerical prediction experimentally

\section{Numerical methods and figures of merit}

Numerical simulations are performed using the Rigorous Coupled Wave Analysis (RCWA) method [1] The global absorptance $A_{g}(\lambda)$ is deduced from the energy conservation law: $A_{g}(\lambda)=1-R(\lambda)-T(\lambda)$

Two figures of merit (FOMs):

$\checkmark$ Integrated quantum efficiency $\boldsymbol{\eta}$ : represents the percentage of incident photons that are absorbed in the whole structure

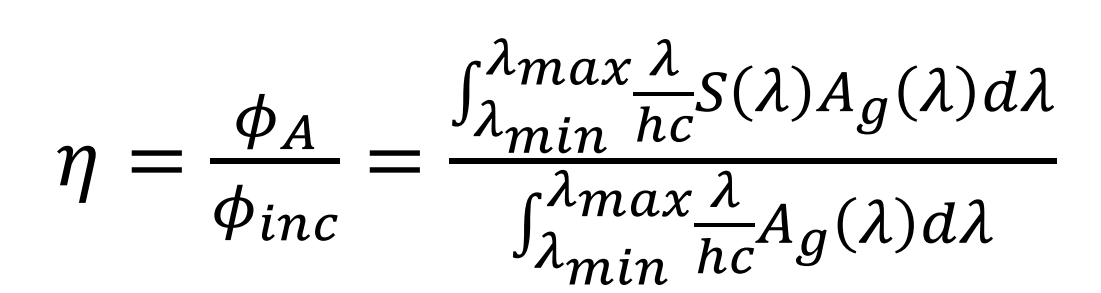

where $\phi_{A}\left(\phi_{i n c}\right)$ is the spectrally integrated absorbed (incident) photon flux, $S(\lambda)$ is the normalized solar spectrum AM1.5G, $\lambda_{\min }=310 \mathrm{~nm}$ and $\lambda_{\max }=800 \mathrm{~nm}$ corresponding respectively to the lower bound of the solar spectrum and to the perovskite band gap.

$\checkmark$ Photonic gain $G_{p h o t}$ : evaluates the enhancement of the generation of photo-electrons due to the photonic structuring in comparison with an equivalent unstructured photonic slab structure

$$
G_{\text {phot }}=\frac{\phi_{3 D}-\phi_{\mathrm{hom}}}{\phi_{\mathrm{hom}}} \times 100
$$

\section{Entire perovskite spheres in an hexagonal array}
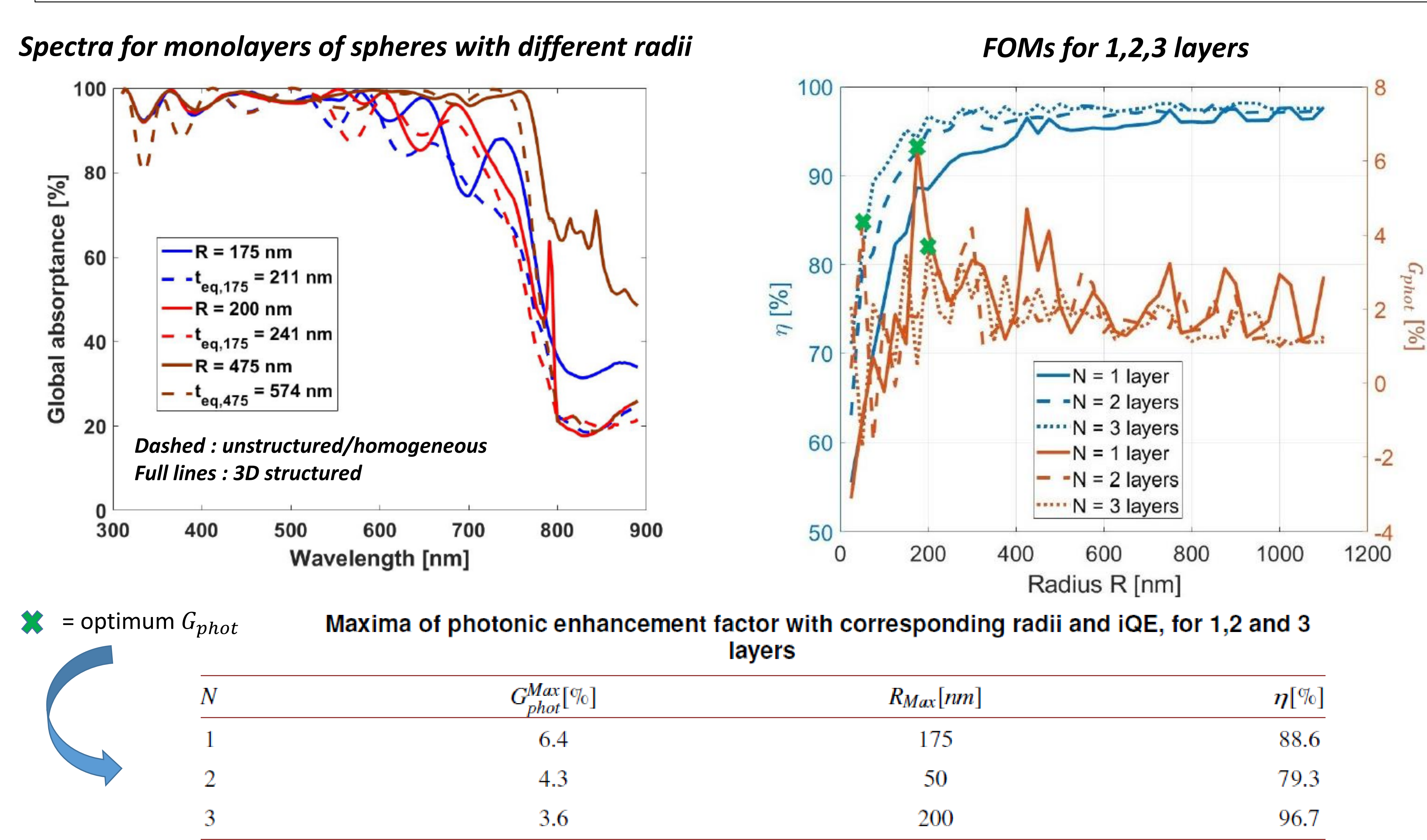
layers
lath

ther with corresponding radii and $\mathrm{QQE}$, for 1,2 and 3

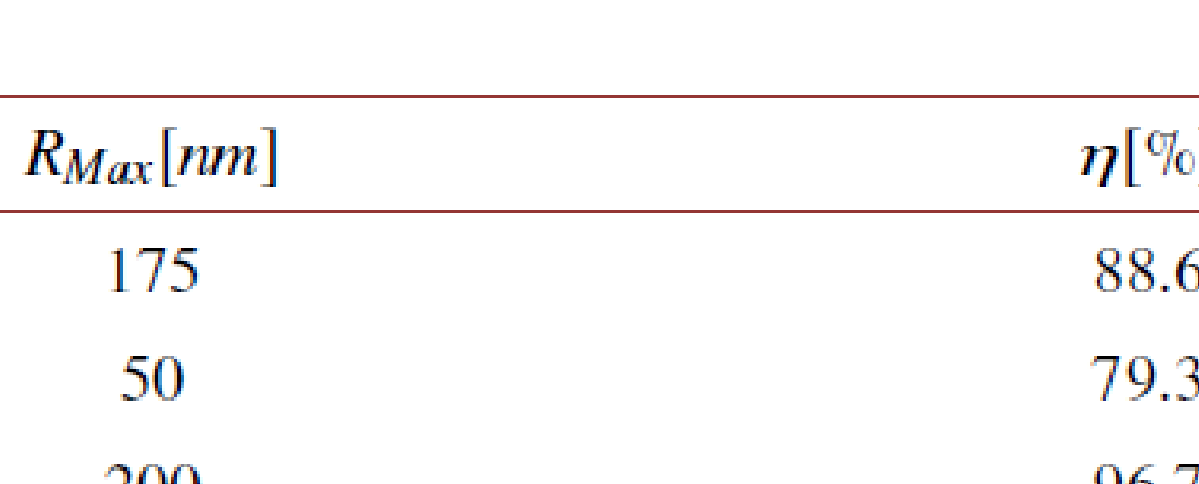

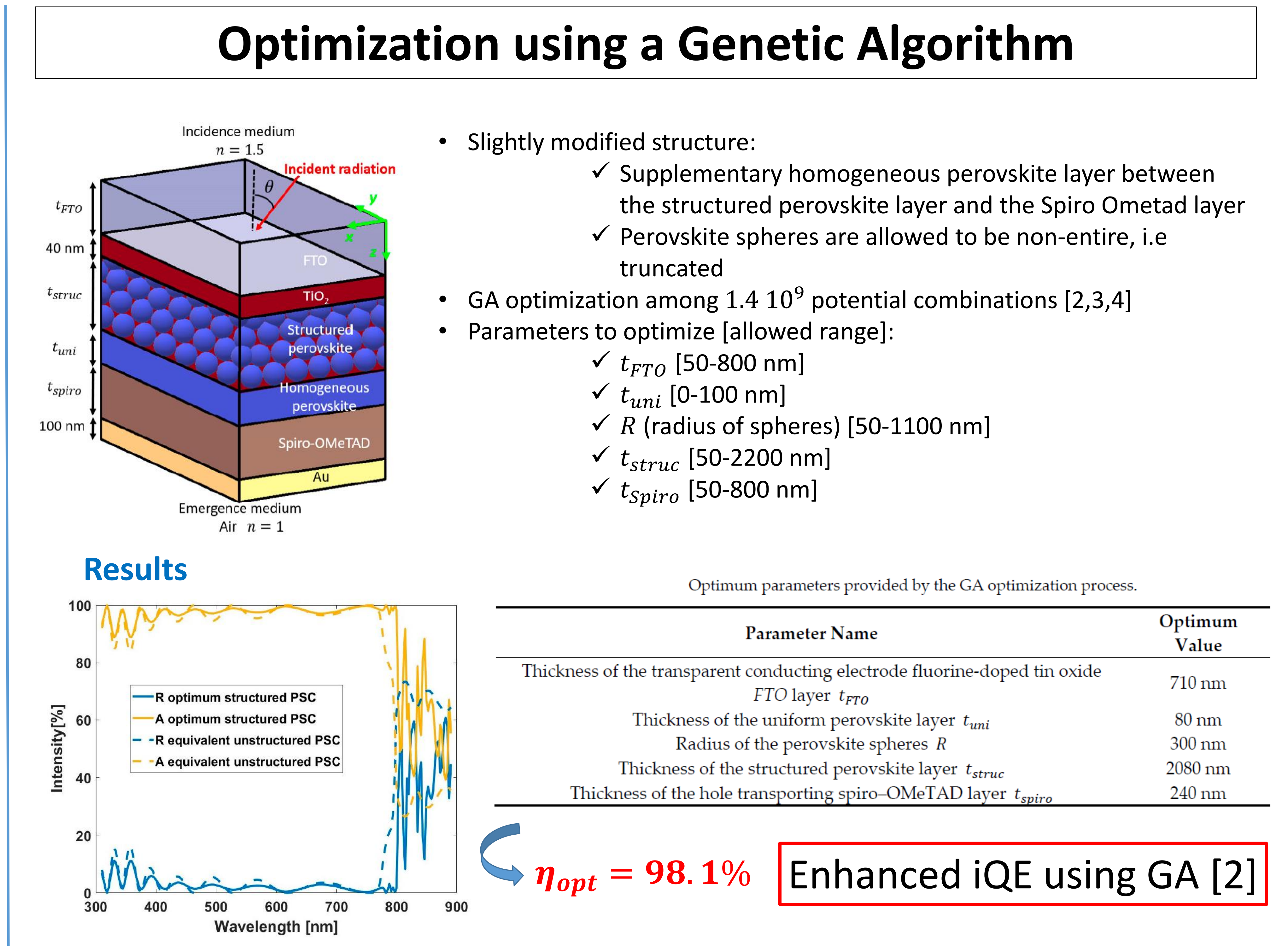

\section{Experimental confirmation}
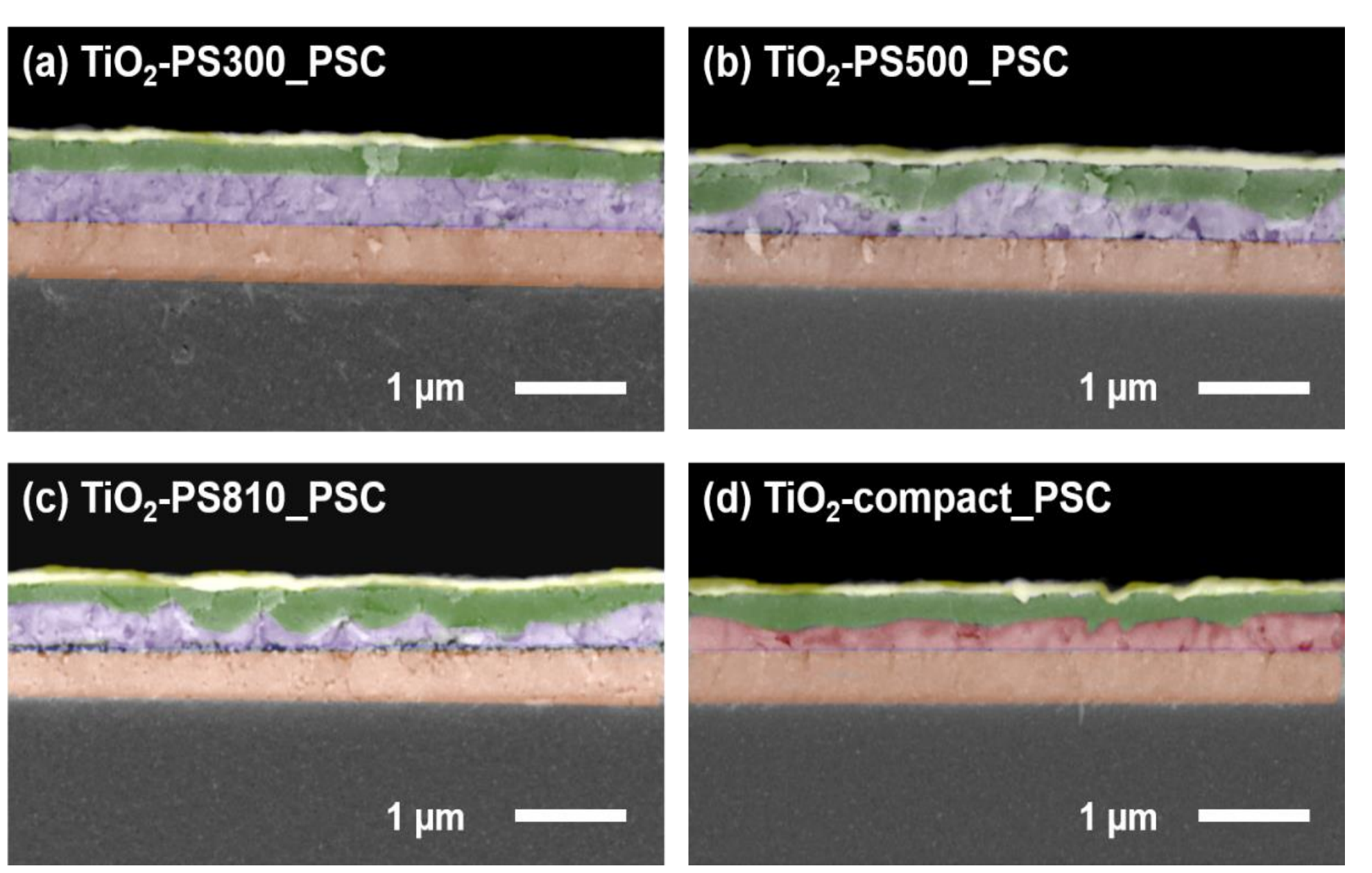

Photonically-structured $\mathrm{TiO}_{2}$ is considered as photoanode layer for perovskite $\mathrm{CH}_{3} \mathrm{NH}_{3} \mathrm{Pbl}_{3}$ solar cells [5]

Three radius are considered $R \sim 110 \mathrm{~nm} ; R \sim 200 \mathrm{~nm} ; R \sim 305 \mathrm{~nm}$ (referred to as PS300, PS500 and PS810 here) and an homogeneous/compact layer for control

(a)
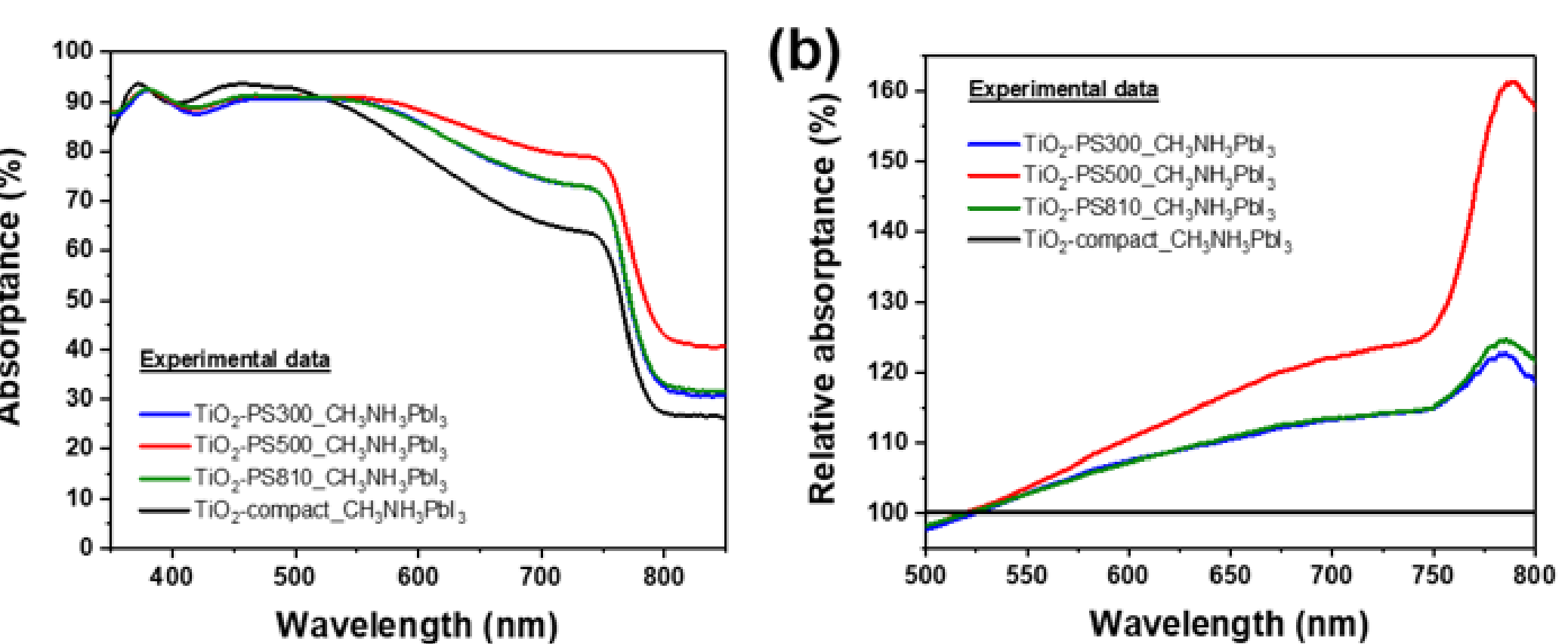

Optimum for $R \sim 200 \mathrm{~nm}$ is found on the absorptance spectra, close to the numerical optimum [1]

Important relative absorptance enhancement close to the electronic bandgap as expected

(c)

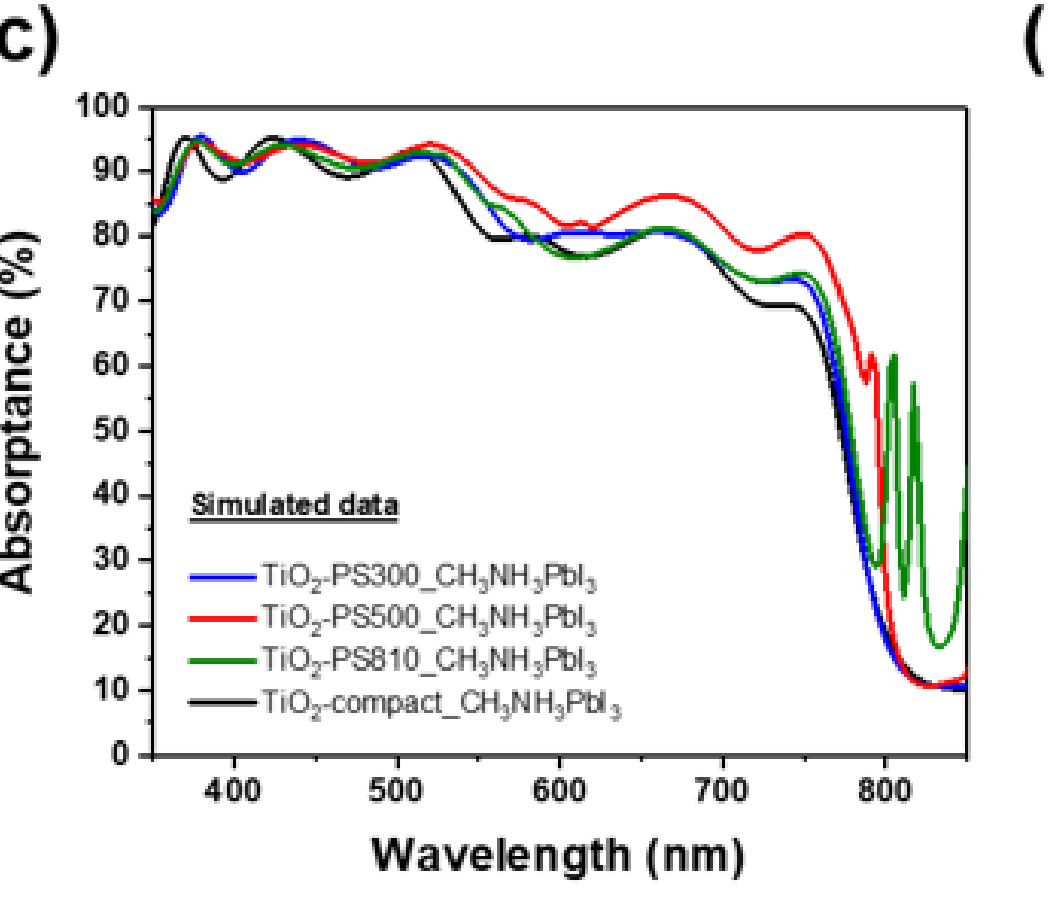

(d)

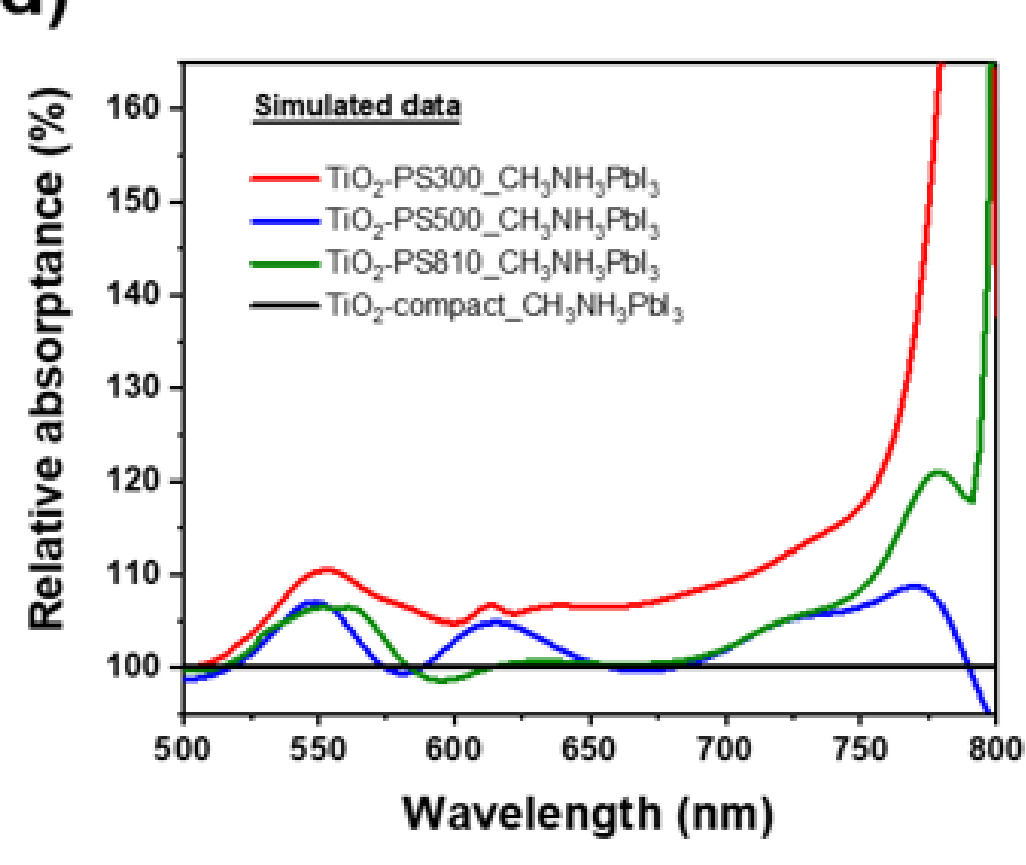

Due to the occurrence of electronic-related counter-effects not taken into account into numerical simualtions, the $R \sim 110 \mathrm{~nm}$ shows an higher power conversion efficiency $(\sim 10 \%)$ than the $R \sim 200 \mathrm{~nm}$ [5].

\section{References}

[1] M. Lobet, P. Piron, J. Dewalque, A.Maho, O. Deparis, C. Henrist, J. Loicq, "Efficiency enhancement of perovskite solar cells based on opal-like photonic crystals," Opt. Express 27, 32308-32322 (2019)

[2] M. Lobet, A. Mayer, A. Maho, P. Piron, J. Dewalque, C. Henrist, J. Loicq, "Opal-Like Photonic Structuring of Perovskite Solar Cells Using a Genetic Algorithm Approach", Appl. Sci., 10, 1783 (2020)

3] A. Mayer, M. Lobet, UV to near-infrared broadband pyramidal absorbers via a genetic algorithm optimization approach. Proc. SPIE, 10671, 1067127-1-11 (2018)

[4] A. Mayer, M. Lobet, A genetic algorithm for addressing computationally expensive optimization problems in optical engineering. Jordan J. Phys., 12, 17-36 (2019)

[5] A.Maho, M. Lobet, N. Daem, P. Piron, G. Spronck, J. Loicq, R. Cloots, P. Colson, C. Henrist, J. Dewalque, Photonic structuration of hybrid inverse-opal TiO2 - perovskite solar cells for enhanced light absorption submitted 